\title{
PARTICLE FILTERING FOR QUANTIZED INNOVATIONS
}

\author{
Ravi Teja Sukhavasi, Babak Hassibi \\ Department of Electrical Engineering \\ California Institute of Technology \\ 1200 E California blvd, Pasadena, CA, 91125
}

\begin{abstract}
In this paper, we re-examine the recently proposed distributed state estimators based on quantized innovations. It is widely believed that the error covariance of the Quantized Innovation Kalman filter [1,2] follows a modified Riccati recursion. We present stable linear dynamical systems for which this is violated and the filter diverges. We propose a Particle Filter that approximates the optimal nonlinear filter and observe that the error covariance of the Particle Filter follows the modified Riccati recursion of [1]. We also simulate a Posterior CramerRao bound (PCRB) for this filtering problem.
\end{abstract}

Index Terms - Distributed state estimation, Sign of Innovation, Particle Filter, Wireless sensor network, Posterior Cramer-Rao bound (PCRB).

\section{INTRODUCTION}

Recent advances in very large-scale integration and microelectromechanical system technology have led to the availability of cheap, low quality and low power consumption sensors in the market. This generated a great deal of interest in wireless sensor networks (WSNs) due to their potential applications in several diverse fields [3]. Sensor network constraints such as limited bandwidth and power inspired a considerable amount of research in developing energy efficient algorithms for network coverage and decentralized detection and estimation using quantized sensor observations [4-6].

Quantizing sensor observations can lead to large quantization noises when the observed values are large which then leads to poor estimation accuracy. In [1], this limitation is overcome by developing an elegant distributed estimation approach based on quantizing the innovation to one bit (so called sign of innovation or SOI). In [2], this is generalized to handle multiple quantization levels. In both cases, it is assumed that the innovation is approximately Gaussian leading to a linear filter and very simple characterization of its error performance. Under the Gaussian assumption, the error covariance matrix associated with the state estimation error satisfies a modified Riccati recursion of the type that appears in [7]. The only difference between this modified Riccati and the traditional one is a scaling factor $\lambda$ multiplying the nonlinear term of the recursion. For the SOI Kalman filter (SOI-KF), $\lambda$ is $\frac{\pi}{2}$ while [2] presents a formula for $\lambda$ in the case of multiple quantization levels. Henceforth, these filters will be referred to as SOI-KF and MLQ-KF, and their associated Riccati recursions as SOI-Riccati and MLQ-Riccati respectively.

For linear time invariant dynamical systems, if Gaussian assumption were realistic, convergence of the modified Riccati must mean the convergence of the corresponding linear filters. Using results presented in [7] one can come up with linear time invariant systems for which the MLQ-Riccati and SOI-Riccati converge. But simulations show that the actual filters do not. This leads one to investigate the relation between the modified Riccati recursions derived in $[1,2]$ and the actual error performance of the filters. In this paper, we precisely try to answer this question. We present simulation results which seem to indicate that the optimal nonlinear filter (approximated by a Particle filter [8-10]) obeys the modified Riccatis of $[1,2]$ while the SOI-KF and MLQ-KF diverge. This is quite surprising in that the Riccatis which are a pure artifact of the Gaussianity assumption predict the performance of the optimal nonlinear filter even when the Gaussian assumption clearly does not hold. We also present a lower bound on the performance of the optimal nonlinear filter. The bound takes the form of a Riccati and is derived using a posterior Cramer-Rao bound developed in [11]. The next section introduces the problem setup and notation.

\section{PROBLEM STATEMENT AND PRELIMINARIES}

Consider the linear dynamical system:

$$
\begin{aligned}
x(n+1) & =A(n) x(n)+w(n) \\
y(n) & =h(n) x(n)+v(n)
\end{aligned}
$$

where $x(n) \in \mathbb{R}^{d}$ is the state, $y(n) \in \mathbb{R}$ is the observation, and $w(n) \in \mathbb{R}^{d}$ and $v(n) \in \mathbb{R}$ are uncorrelated Gaussian white noises with zero means and covariances $Q(n)$ and $R(n) \triangleq \sigma_{v}^{2}(n)$, respectively. The initial state, $x(0)$, of the system, is uncorrelated with both $w(n)$ and $v(n)$.

We use the same problem setup as that used in $[1,2]$. We consider the sensor network configuration in which the fusion center has sufficient power to broadcast its predicted output 
and the corresponding error covariance to its sensors. Sensors are assumed to have limited power and hence their transmission of information should be limited. Here, we assume that the energy required for receiving messages is much less than that for transmitting.

Once a scheduling algorithm is in place, at each time instant, a sensor makes a measurement $y(n)$ and computes the innovation $\epsilon(n)=y(n)-\hat{y}(n \mid n-1)$, where $\hat{y}(n \mid n-1)=h \hat{x}(n \mid n-1)$ together with the variance of the innovation $\sigma_{\epsilon}^{2}(n)=h(n) P(n \mid n-1) h^{T}(n)+R(n)$ are received by the sensor from the fusion center with $\hat{x}(n \mid n-1)$ being the one step predictor of the state. $[1,2]$ propose methods to quantize $\epsilon(n)$ and use the quantized innovations to update the state estimate.

Though simple and elegant, the filters developed in $[1,2]$ can cause the state estimate error to diverge as will be shown shortly using examples. We then propose a Particle filter algorithm to approximate the optimal nonlinear filter and surprisingly, we observe that its error covariance follows the modified Riccati recursion developed in $[1,2]$.

\section{BACKGROUND}

Let the normalized innovation, $\bar{\epsilon}(n)=\frac{\epsilon(n)}{\sigma_{\epsilon}}$, be quantized as

$$
b(n)=\left\{\begin{array}{lr}
0 & -z_{1}<\bar{\epsilon}(n) \leq z_{1} \\
\operatorname{Sign}(\bar{\epsilon}(n)) z_{i} & z_{i}<|\bar{\epsilon}(n)| \leq z_{i+1}
\end{array}\right.
$$

with the convention that $z_{N+1}=\infty$. Then the multiple level quantized Kalman filter (MLQ-KF) of [2] can be expressed as follows

$$
\begin{gathered}
\hat{x}(n \mid n)=\hat{x}(n \mid n-1)+\frac{f_{N}(n) P(n \mid n-1) h^{T}(n)}{\sqrt{h P(n \mid n-1) h^{T}(n)+R(n)}} \\
P(n \mid n)= \\
P(n \mid n-1)-2 \sum_{k=1}^{N} \frac{\left(\phi\left(z_{k}\right)-\phi\left(z_{k+1}\right)\right)^{2}}{Q\left(z_{k}\right)-Q\left(z_{k+1}\right)} \\
\times \frac{P(n \mid n-1) h^{T}(n) h(n) P(n \mid n-1)}{h(n) P(n \mid n-1) h^{T}(n)+R(n)}
\end{gathered}
$$

where

$$
\begin{aligned}
f_{N}(n) & =\sum_{k=1}^{N} \operatorname{Sign}(b(n)) I_{k}(b(n)) \frac{\phi\left(z_{k}\right)-\phi\left(z_{k+1}\right)}{Q\left(z_{k}\right)-Q\left(z_{k+1}\right)} \\
\phi(x) & =\frac{1}{\sqrt{\pi}} \exp \left(-\frac{x^{2}}{2}\right), Q(x)=\int_{x}^{\infty} \phi(x) d x
\end{aligned}
$$

One can recover the Sign of innovation Kalman filter (SOI$\mathrm{KF}$ ) of [1] by setting $N=1$ and $z_{1}=0$.

\section{PARTICLE FILTER}

The Particle filter here adopted to using signs of innovations $\{b(n)\}$ to estimate the state vector, is given by

\section{Alg. Particle Filter}

1. Set $n=0$. For $i=1, \cdots, N$, initialize the particles, $x^{i}(0 \mid-1) \sim P_{0}\left(x_{0}\right)$ and set $\hat{x}(0 \mid-1)=[0, \cdots, 0]^{T}$

2. At time $n$, set $b(n)=\operatorname{Sign}(y(n)-h \hat{x}(n \mid n-1))$.

3. Calculate the importance weights,

$$
w^{i}(n)=Q\left(-b(n) \frac{h\left(x^{i}(n \mid n-1)-\hat{x}(n \mid n-1)\right)}{\sigma_{v}(n)}\right)
$$

4. Measurement update

$$
\hat{x}(n \mid n)=\frac{1}{N} \sum_{i=1}^{N} w^{i}(n) x^{i}(n \mid n-1)
$$

5. Resample $N$ particles with replacement accoding to,

$$
\operatorname{Prob}\left(x^{i}(n \mid n)=x^{j}(n \mid n-1)\right)=\bar{w}^{j}(n \mid n-1)
$$

where the normalized weights are given by

$$
\bar{w}^{j}(n \mid n-1)=\frac{w^{j}(n \mid n-1)}{\sum_{i=1}^{N} w^{i}(n \mid n-1)}
$$

6. For $i=1, \cdots, N$, predict new particles according to,

$$
x^{i}(n+1 \mid n) \sim p\left(x_{n+1} \mid x^{i}(n \mid n)\right)
$$

7. Set $\hat{x}(n+1 \mid n)=A(n) \hat{x}(n \mid n)$. Also, set $n=n+1$ and iterate from step 2 .

Step 3 is obtained as follows, .

$$
\begin{array}{r}
b(n)=\operatorname{Sign}(h x(n)+v(n)-h \hat{x}(n \mid n-1)) \\
w^{i}(n)=\operatorname{Prob}(b(n)= \pm 1 \mid x(n), b(0: n-1)) \\
=\operatorname{Prob}(v(n) \gtrless-h(x(n)-\hat{x}(n \mid n-1))) \\
=Q\left(\mp \frac{h\left(x^{i}(n \mid n-1)-\hat{x}(n \mid n-1)\right)}{\sigma_{v}(n)}\right)
\end{array}
$$

The above algorithm can be trivially adapted to handle the case of multiple quantization levels. Note that the algorithm can be optimized in a number of ways to use far fewer particles. But, in this work, we are only interested in using Particle filtering to approximate the optimal nonlinear filter.

\section{COUNTER-EXAMPLES}

We present two examples in this section.

1. A stable linear system for which both the MLQ-KF and SOI-KF diverge. 
2. A stable linear system for which the error covariance of SOI-KF seems to converge but does not follow the SOI-Riccati.

Interesting thing about these examples is that the Particle filter converges and follows the SOI-Riccati while SOI-KF does not. Monte Carlo simulations of the error variance of the SOIKF, MLQ-KF and the Particle filter are shown in Fig. 1 and Fig. 2. In both figures, the $x$-axis denotes time $n$ and the $y$-axis denotes $E\|x(n)-\hat{x}(n \mid n)\|^{2}=\operatorname{trace}(P(n \mid n))$. The filter used to obtain $\hat{x}(n \mid n)$ is evident from the context.

\subsection{Example 1}

Consider a linear time invariant system of the form (1) with the following parameters: $A=\left(\begin{array}{ccc}0.95 & 1 & 0 \\ 0 & 0.9 & 10 \\ 0 & 0 & 0.95\end{array}\right), Q=$ $2 \mathcal{I}_{3}, h=\left(\begin{array}{lll}1 & 0 & 2\end{array}\right), R \triangleq \sigma_{v}^{2}=2.5$ and $P_{0}=0.01 \mathcal{I}_{3}$, where $\mathcal{I}_{m}$ denotes an $m \times m$ identity matrix. Note that the eigen values of $A,(0.95,0.9,0.95)$ are all less than 1. Fig. 1(a) compares the Monte Carlo simulations of the SOI-KF and Particle fiter with the SOI-Riccati. It also shows the posterior CramerRao lower bound for this problem, the details of which will be discussed in Section 6. The Riccati recursion associated with the full information Kalman filter is also plotted for comparison. Fig 1(b) shows divergence of the MLQ-KF with 4 levels of quantization $\left(-z_{2},-z_{1}, z_{1}, z_{2}\right)$ where $z_{1}=0.38$ and $z_{2}=1.24$.

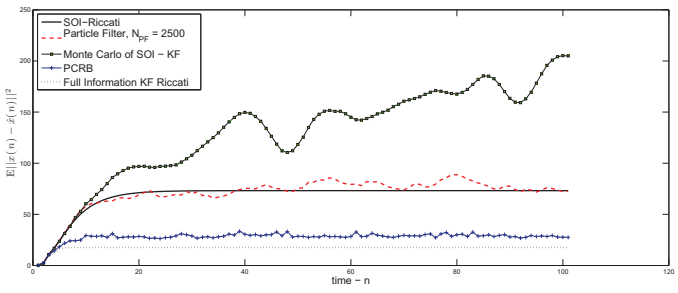

(a) SOI-KF and Particle filter

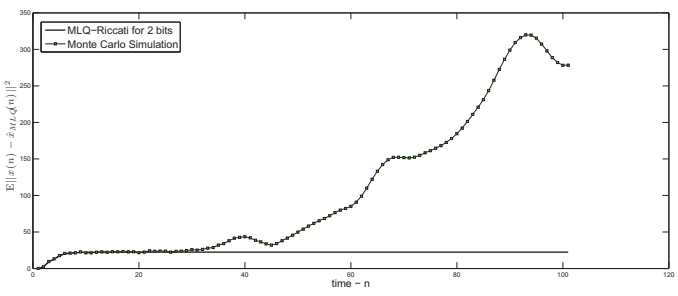

(b) MLQ-KF with 4 levels of quantization

Fig. 1. In (a), SOI-KF clearly diverges while Particle filter converges to the SOI-Riccati. Posterior Cramer-Rao bound is also shown. From (b), MLQ-KF with 4 levels of quantization also diverges. Experiments show that MLQ-KF diverges with 6 levels of qantization as well but converges to MLQ-Riccati with 8 levels.

\subsection{Example 2}

Consider the following set of parameters

$A=\left(\begin{array}{cccc}0.95 & 1 & 0 & 0 \\ 0 & 0.9 & 7 & 0 \\ 0 & 0 & 0.6 & 2 \\ 0 & 0 & 0 & 0.95\end{array}\right), Q=2 \mathcal{I}_{4}, h=\left(\begin{array}{llll}1 & 0 & 1 & 0\end{array}\right)$, $R \triangleq \sigma_{v}^{2}=2.5$ and $P_{0}=0.01 \mathcal{I}_{4}$. In this case too, note that $A$ is stable. Fig. 2(a) compares the Monte Carlo simulations of SOI-KF and Particle fiter with the SOI-Riccati. The plot indicates that the SOI-KF doesn't diverge for this problem but it doesn't follow the SOI-Riccati while the Particle filter does. Fig 2(b) shows divergence of the MLQ-KF with 4 levels of quantization as mentioned above.

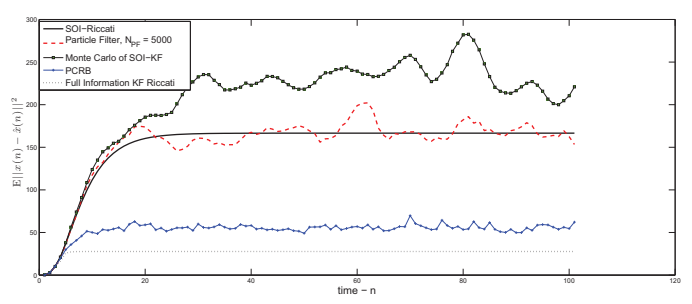

(a) SOI-KF and Particle filter

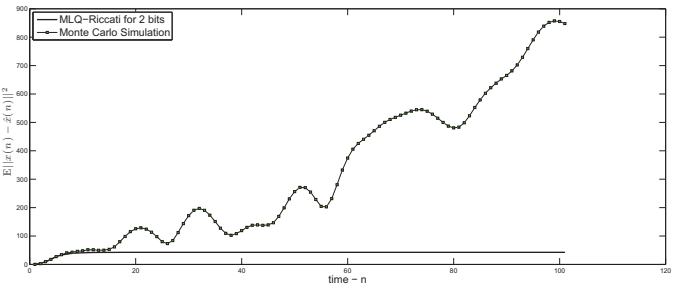

(b) MLQ-KF with 4 levels of quantization

Fig. 2. In (a), SOI-KF seems to converge but doesn't follow SOIRiccati while Particle filter converges to the SOI-Riccati. Posterior Cramer-Rao bound is also shown. From (b), MLQ-KF with 4 levels of quantization diverges. Experiments show that MLQ-KF with 6, 8 and 10 levels of quantization diverge too.

\section{POSTERIOR CRAMER RAO BOUND}

Since we are interested in estimating the state using the signs of innovations, we treat $\mathbf{b}_{n} \triangleq b(0: n)$ as the vector of measured data. Let $p\left(x(n), \mathbf{b}_{n}\right)$ be the joint probability desnity of the pair $\left(x(n), \mathbf{b}_{n}\right)$, and let $g\left(\mathbf{b}_{n}\right)$ be a function of $\mathbf{b}_{n}$, which is an estimate of $x(n)$. The PCRB on the estimation error has the form

$$
P(n \mid n) \triangleq E\left\{\left(g\left(\mathbf{b}_{n}\right)-x(n)\right)\left(g\left(\mathbf{b}_{n}\right)-x(n)\right)^{T}\right\} \geq J_{n}^{-1}
$$


where $J_{n}$ is the $d \times d$ Fisher information matrix with the elements

$$
J_{n}(i, j)=E\left(-\frac{\partial^{2} \log p\left(x(n), \mathbf{b}_{n}\right)}{\partial x_{i}(n) \partial x_{j}(n)}\right) \quad i, j=1, \cdots, d
$$

where $x_{i}(n)$ is the $i^{\text {th }}$ component of the $d$-dimensional vector $x(n)$. Using the notation $\nabla \Theta=\left[\frac{\partial}{\partial \Theta_{1}}, \cdots, \frac{\partial}{\partial \Theta_{r}}\right]^{T}, \Delta_{\Psi}^{\Theta}=$ $\nabla \Psi \nabla_{\Theta}^{T}$ we can write

$$
J_{n}=E\left\{-\Delta_{x(n)}^{x(n)} \log p\left(x(n), \mathbf{b}_{n}\right)\right\}
$$

It was shown in [11] that $J_{n}$ satisfies the following recursion

$$
J_{n+1}=D_{n}^{22}-D_{n}^{21}\left(J_{n}+D_{n}^{11}\right)^{-1} D_{n}^{12}
$$

where

$$
\begin{aligned}
& D_{n}^{11}=A^{T}(n) Q(n)^{-1} A(n), D_{n}^{12}=-A^{T}(n) Q(n)^{-1}=\left[D_{n}^{21}\right]^{T} \\
& D_{n}^{22}=Q^{-1}+E\left\{-\Delta_{x(n+1)}^{x(n+1)} \log p\left(b(n+1) \mid x(n+1), \mathbf{b}_{n}\right)\right\}
\end{aligned}
$$

Consider the second expression in (8). From (5) we have

$$
p\left(b(n+1) \mid x(n), \mathbf{b}_{n}\right)=Q\left(-\frac{b(n+1) h(x(n+1)-\hat{x}(n+1 \mid n))}{\sigma_{v}}\right)
$$

where $\hat{x}(n+1 \mid n)=E\left(x(n+1) \mid \mathbf{b}_{n}\right)$. Upon straightforward differentiation, we get

$$
\begin{array}{r}
-\Delta_{x(n+1)}^{x(n+1)} \log p\left(b(n+1) \mid x(n+1), \mathbf{b}_{n}\right) \\
=\frac{h^{T} h}{\sigma_{v}^{2}} \underbrace{\left(z_{n+1} \frac{\phi\left(z_{n+1}\right)}{Q\left(-z_{n+1}\right)}+\left(\frac{\phi\left(z_{n+1}\right)}{Q\left(-z_{n+1}\right)}\right)^{2}\right)}_{\alpha\left(z_{n+1}\right)}
\end{array}
$$

where $z_{n+1}=\frac{b(n+1) h(x(n+1)-\hat{x}(n+1 \mid n))}{\sigma_{v}}$. Define $\alpha_{n+1}=$ $E\left(\alpha\left(z_{n+1}\right)\right)$. We can then write (7) as follows

$$
J_{n+1}=Q^{-1}+\frac{h^{T} \alpha_{n+1} h}{\sigma_{v}^{2}}-Q^{-1} A^{T}\left(J_{n}+A Q^{-1} A^{T}\right)^{-1} A Q^{-1}
$$

Applying matrix inversion lemma, we get

$$
\left(Q^{-1}-Q^{-1} A\left(J_{n}+A^{T} Q^{-1} A\right)^{-1} A^{T} Q^{-1}\right)^{-1}=A J_{n}^{-1} A^{T}+Q
$$

Applying matrix inversion lemma again to (10) and using (11), we get

$$
J_{n+1}^{-1}=A J_{n}^{-1} A^{T}+Q-\frac{\left(A J_{n}^{-1} A^{T}+Q\right) h^{T} h\left(A J_{n}^{-1} A^{T}+Q\right)}{\frac{\sigma_{v}^{2}}{\alpha_{n+1}}+h\left(A J_{n}^{-1} A^{T}+Q\right) h^{T}}
$$

Note that this has the form of the traditional Riccati recursion. From its structure, it is easy to see that it predicts the error performance of the full information Kalman filter applied to a modified system whose observation noise variance at time $n-$ 1 is scaled by a factor of $\frac{1}{\alpha_{n}}$ compared to the original. Simple analysis shows that the function $\alpha(z) \in(0,1) \forall z \in \mathbb{R}$. Hence, $\alpha_{n}$ which is the average of $\alpha\left(z_{n}\right)$, defined in (9), also lies in $(0,1)$. So, scaling by $\frac{1}{\alpha_{n}}$ only increases observation noise. Hence the Riccati obtained above is strictly bounded below by the error performance of the full information Kalman filter applied to the original system. Though (12) is a non-trivial bound, it is not analytically tractable to compute $\alpha_{n}$. Monte Carlo simulations of (12) are presented in Fig. 1 and Fig. 2.

\section{CONCLUSIONS}

We demonstrated that, contrary to conventional wisdom, the error performance of the SOI-KF and MLQ-KF do not generally follow the modified Riccati recursions developed in $[1,2]$. Surprisingly, simulations seem to suggest that these modified Riccatis predict the error performance of the optimal nonliear mean-square error filter (which we have here approximated by a Particle filter). Demonstrating whether this is truly the case or not seems worthwhile of further scrunity.

\section{REFERENCES}

[1] A. Ribeiro, G. B. Giannakis, and S. I. Roumeliotis, "Soi-kf: Distributed kalman filtering with low-cost communications using the sign of innovations," IEEE Transactions on Signal Processing, vol. 54, no. 12, pp. 4782-4795, 2006.

[2] Keyou You, Lihua Xie, Shuli Sun, and Wendong Xiao, "Multiple-level quantized innovation kalman filter," in 17th International Federation of Automatic Control. 2008, Seoul, Korea.

[3] I.F. Akyildiz, Weilian Su, Y. Sankarasubramaniam, and E. Cayirci, "A survey on sensor networks," Communications Magazine, IEEE, vol. 40, no. 8, pp. 102-114, 2002.

[4] A. Krasnopeev, Jin-Jun Xiao, and Zhi-Quan Luo, "Minimum energy decentralized estimation in sensor network with correlated sensor noise," in ICASSP, 2005, vol. 3, pp. iii/673-iii/676 Vol. 3.

[5] Zhi-Quan Luo and Jin-Jun Xiao, "Universal decentralized estimation in a bandwidth constrained sensor network," in ICASSP, 2005, vol. 4, pp. iv/829-iv/832 Vol. 4.

[6] G Rickard Karlsson and Fredrik Gustafsson, "Particle filtering for quantized sensor information," in 13th European Signal Processing Conference, EUSIPCO. 2005, EURASIP, Turkey.

[7] B. Sinopoli, L. Schenato, M. Franceschetti, K. Poolla, M.I. Jordan, and S.S. Sastry, "Kalman filtering with intermittent observations," in Proceedings. 42nd IEEE Conference on Decision and Control, 2003, 2003, vol. 1, pp. 701-708 Vol.1.

[8] N.J. Gordon, D.J. Salmond, and A.F.M. Smith, "Novel approach to nonlinear/non-gaussian bayesian state estimation," Radar and Signal Processing, IEE Proceedings-F, vol. 140, no. 2, pp. 107-113, 1993.

[9] D. Crisan and A. Doucet, "A survey of convergence results on particle filtering methods for practitioners," IEEE Transactions on Signal Processing, vol. 50, no. 3, pp. 736-746, 2002.

[10] M.S. Arulampalam, S. Maskell, N. Gordon, and T. Clapp, "A tutorial on particle filters for online nonlinear/non-gaussian bayesian tracking," IEEE Transactions on Signal Processing, vol. 50, no. 2, pp. 174-188, 2002.

[11] P. Tichavsky, C.H. Muravchik, and A. Nehorai, "Posterior cramer-rao bounds for discrete-time nonlinear filtering," IEEE Transactions on Signal Processing, vol. 46, no. 5, pp. 13861396, 1998. 